\title{
A Circularly Polarized Short Backfire Antenna Excited by an Unbalance-Fed Cross Aperture
}

\author{
RongLin Li, Senior Member, IEEE, Dane C. Thompson, Student Member, IEEE, \\ John Papapolymerou, Senior Member, IEEE, Joy Laskar, Fellow, IEEE, and \\ Manos M. Tentzeris, Senior Member, IEEE
}

\begin{abstract}
An unbalance-fed cross aperture is developed to excite a short backfire antenna (SBA) for circular polarization. The cross aperture consists of two orthogonal $\mathrm{H}$-shaped slots with a pair of capacitive stubs and is fed by a single probe that forms an unbalanced feed with a shorting pin. It is demonstrated that the cross-aperture-excited SBA can achieve an axial ratio $(\leqslant 3 \mathrm{~dB})$ bandwidth of $4.2 \%$ with a voltage standing wave ratio (VSWR) bandwidth of $6.5 \%$ (VSWR $<1.2$ ) and a gain of $14 \mathrm{dBi}$. The antenna structure is described and the simulation and experimental results are presented. The mechanisms for impedance matching and circular-polarization production are analyzed.
\end{abstract}

Index Terms-Circularly polarized antenna, short backfire antenna (SBA), slot-fed antenna.

\section{INTRODUCTION}

$\mathbf{T}$ HE short backfire antenna (SBA) has been widely used in mobile/maritime satellite communications, tracking, telemetry, and wireless applications due to its high gain, low sidelobe level, and simple configuration [1]-[3]. For satellite communications, it is desirable to employ a circularly polarized antenna to avoid the power loss caused by Faraday polarization rotation. To achieve circular polarization, the most popular excitation structure for the SBA is a cross dipole that consists of two orthogonal dipoles. Usually the cross dipole needs an external polarizer such as a $90^{\circ}$ hybrid coupler for circular polarization [4]. Even though the cross dipole can also produce circular polarization without the external hybrid component by the self-phasing, a balun circuit is still required for the cross dipole [5]. The cross-dipole-excited SBA also has a narrow frequency bandwidth for its input impedance because the SBA is essentially a leaky cavity structure. A nature impedance match bandwidth is only $3-5 \%$ for a $1.5: 1$ voltage standing wave ratio (VSWR) [6].

Another well known excitation topology for circular polarization is the cross aperture which consists of two orthogonal rectangular slots and has been widely used to excite microstrip antennas [7]-[10]. The circular polarization is generated by building two rectangular slots with slightly different lengths so that the radiation from one slot is $90^{\circ}$ out of phase with the other. When the radiated fields from two orthogonal slots

Manuscript received January 13, 2005; revised October 19, 2005. This work was supported in part by the Georgia Electronic Design Center (GEDC), in part by the NSF under CAREER Award ECS-9984761 and Grant ECS-0313951, and in part by the NSF Packaging Research Center.

The authors are with the Georgia Electronic Design Center, School of Electrical and Computer Engineering, Georgia Institute of Technology, Atlanta, GA 30332-0250 USA (e-mail: rlli@ece.gatech.edu).

Digital Object Identifier 10.1109/TAP.2006.869910 combine in the far zone with $90^{\circ}$ phase difference, they produce circular polarization at broadside. The cross aperture is usually fed by a microstrip line [7]-[9] or by a single probe [10]. Unfortunately, neither the microstrip-line feed nor the single-probe feed could be directly applied to the SBA because the excitation structure for a SBA must be placed approximately a quarter-wavelength above the primary reflector (or the ground plane) to excite the leaky waves needed for the backfire radiation. Obviously the microstrip feed is not suitable for this situation. If a single-probe feed is adopted, it would cause a significant inductive component in the input impedance due to the parasitic inductance from the long feed probe (about a quarter-wavelength), thus leading to a difficulty in impedance matching. Furthermore, a feed probe of one quarter-wavelength itself acts as a resonant monopole antenna which could radiate a strong cross-polarized component, considerably degrading the radiation performance of the SBA.

Before a cross aperture could be employed to excite the SBA, there is an additional difficulty that needs to be overcome. To achieve circular polarization, one of the two orthogonal slots of the cross aperture has to be capacitive if the other is inductive. For a rectangular slot, its length must be longer than a half wavelength to make it capacitive [10]. As a result, the size of the patch on which the cross aperture is etched must be much larger than a half wavelength. Note that the diameter of the subreflector of an SBA is usually less than a half wavelength. Therefore the excitation structure would be much larger than the subreflector, thus blocking the reflection from the primary reflector and changing the desirable radiation performance of the SBA.

Recently, an unbalance-fed H-shaped slot has been developed for a linearly polarized SBA [11]. In this paper, an unbalance-fed cross aperture is proposed to excite the SBA for circular polarization. The unbalanced feed formed by a feed probe and a shorting pin serves as a two-wire transmission line and as an impedance matching circuit, thus alleviating the negative influence of a single feed on the radiation pattern and realizing an excellent impedance matching. The cross aperture consists of two orthogonal $\mathrm{H}$-shaped slots with different lengths. (The H-shaped configuration can increase the effective length of a slot.) A pair of short stubs is introduced inside the longer $\mathrm{H}$-shaped slot to make it more capacitive, allowing a further decrease in the size of the cross aperture. It will be shown that the $\mathrm{H}$-shaped slots and the short stubs are necessary for the production of circular polarization. In the next section, the antenna structure is described. Then simulation and experimental results are presented. Finally the mechanisms for circular polarization and impedance matching are investigated. 


\section{ANTENNA STRUCTURE}

The configuration of the cross-aperture-excited circularly polarized SBA is illustrated in Fig. 1. The SBA contains a pair of circular reflectors (i.e., a primary reflector and a subreflector), a circular rim, and an excitation structure that consists of a cross aperture fed by a single probe with a shorting pin. The feed probe (extended from an SMA connector) and the shorting pin (connected to the primary reflector) forms an unbalanced feed which is located at the center of the SBA and is aligned along the diagonal of the cross aperture. The cross aperture consists of two orthogonal H-shaped slots (the longer one is called "Slot $\mathrm{X}$ " and the shorter one "Slot Y") etched on a circular patch (called "slotted patch") whose diameter is equal to that of the subreflector. The H-shaped slot is divided into two parts (i.e., a wider part and a narrower part) that play different roles. The purpose of the wider part is to increase the effective length (i.e., the perimeter) of each slot, hence making the slot close to resonance. The longer the effective length, the closer to resonance is the slot; but the maximum width of the wider part is constrained by the size of the slotted patch. The reason for introducing a narrower part in the slots is to excite over the cross aperture a stronger electric-field distribution which is coupled to the reflectors of the SBA. (Simulation shows that the electric-field distribution over the cross aperture is concentrated along the narrower parts of two H-shaped slots.) The area of the narrower part controls how much power could be transferred from the feed probe to the aperture (i.e., the region between the rim and the subreflector) of the SBA, thus affecting the input impedance of the antenna. The length of each slot makes a notable impact on the phase of the radiation field associated with this slot, especially near a resonant point. In order to accomplish a phase difference ( $90^{\circ}$ for perfect circular polarization) between the radiated fields from the two slots, we set Slot $\mathrm{X}$ to be $0.1 \lambda_{0}$ longer than Slot Y. This length difference is not enough for a $90^{\circ}$ phase difference. Therefore a pair of short $\left(<0.1 \lambda_{0}\right)$ stubs connecting respectively to the feed probe and the shorting pin is added to the middle of Slot X. The pair of short stubs can be considered as a capacitor parallel to Slot X, making the slot more capacitive (or look further longer).

The SBA is designed using Micro-Stripes 6.5-a TLM (Transmission-Line Matrix) based full-wave electromagnetic simulator. A prototype is built at $5.8 \mathrm{GHz}$ ISM band (5.7-5.9 GHz). The subreflector and the cross aperture are printed on a $0.508-\mathrm{mm}$ (20 mils) RT/duroid 5880 substrate $\left(\varepsilon_{r}=2.2\right)$ and the printed subreflector is supported by the rim. The optimal values of the geometric parameters of the cross-aperture-excited SBA are listed in Table I. The overall dimensions of the SBA are $0.54 \lambda_{0}\left(\lambda_{0}=\right.$ the free-space wavelength at the design frequency $5.8 \mathrm{GHz}$ ) in height and $1.93 \lambda_{0}$ in diameter, which are close to the typical values $\left(0.5 \lambda_{0}\right.$ in height and $2 \lambda_{0}$ in diameter) for a conventional SBA. The diameter and height of the slotted patch are $0.46 \lambda_{0}$ and $0.27 \lambda_{0}$, respectively. The width of the wider part of the $\mathrm{H}$-shaped slots is chosen to be 6 times the width of the narrower part that is optimized to be $1.0 \mathrm{~mm}$. The total lengths of Slots $\mathrm{X}$ and $\mathrm{Y}$ are respectively $20 \mathrm{~mm}\left(\sim 0.38 \lambda_{0}\right)$ and $15 \mathrm{~mm}\left(\sim 0.28 \lambda_{0}\right)$, both of which are much shorter than a half wavelength. The length and the width of the capacitive stubs are adjusted to $2.5 \mathrm{~mm}$ and $0.3 \mathrm{~mm}$. The diameter and the center-to-center

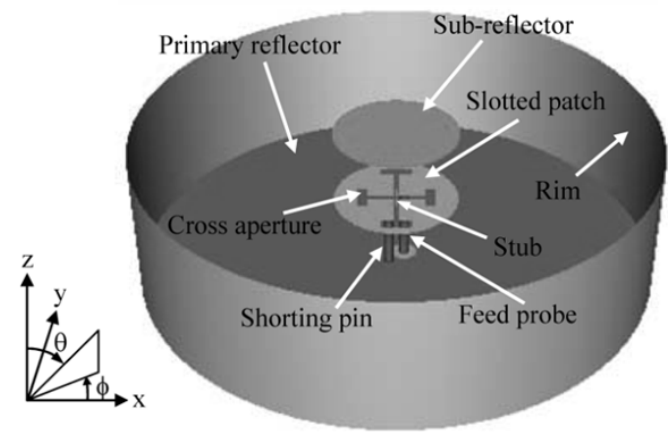

(a)

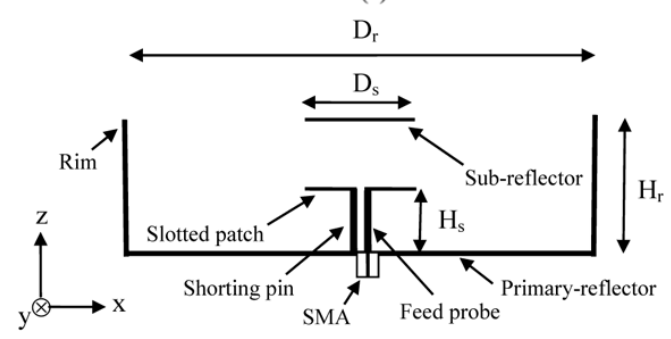

(b)

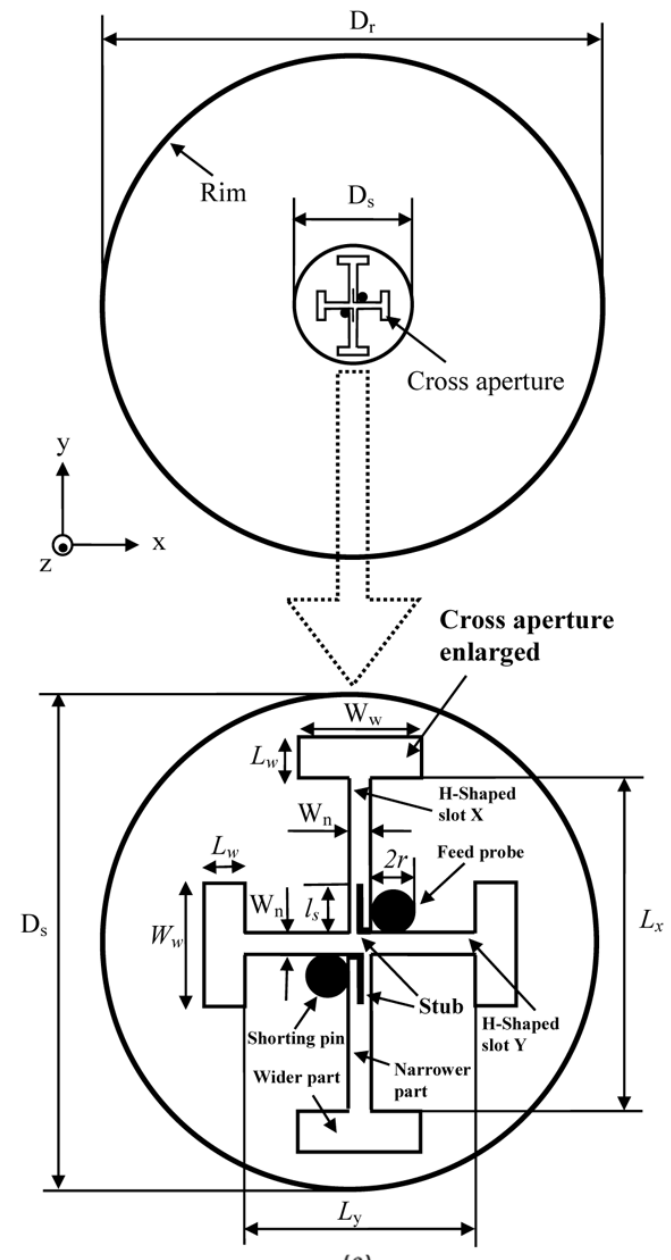

(c)

Fig. 1. A circularly polarized short backfire antenna excited by an unbalance-fed cross aperture. (The subreflector is printed on an RT/duroid substrate that is placed on the rim.) (a) Perspective view. (b) Side view. (c) Top view.

distance of the feed probe and the shorting pine are $2 \mathrm{~mm}$ and $3 \sqrt{2} \mathrm{~mm}$, respectively. All these parameters were optimized 
TABLE I

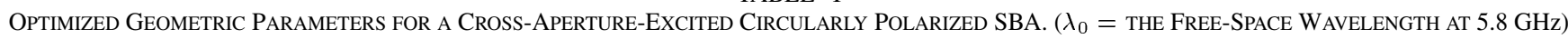

\begin{tabular}{|c|c|c|c|}
\hline $\mathrm{D}_{\mathrm{r}}$ & $100 \mathrm{~mm}\left(1.93 \lambda_{0}\right)$ & $W_{n}$ & $1.0 \mathrm{~mm}\left(0.0193 \lambda_{0}\right)$ \\
\hline $\mathrm{H}_{\mathrm{r}}$ & $28 \mathrm{~mm}\left(0.54 \lambda_{0}\right)$ & $W_{w}$ & $6.0 \mathrm{~mm}\left(0.116 \lambda_{0}\right)$ \\
\hline $\mathrm{D}_{\mathrm{s}}$ & $24 \mathrm{~mm}\left(0.46 \lambda_{0}\right)$ & $L_{w}$ & $2.0 \mathrm{~mm}\left(0.0386 \lambda_{0}\right)$ \\
\hline $\mathrm{H}_{s}$ & $14 \mathrm{~mm}\left(0.27 \lambda_{0}\right)$ & $l_{s}$ & $2.5 \mathrm{~mm}\left(0.048 \lambda_{0}\right)$ \\
\hline $\mathrm{L}_{\mathrm{x}}$ & $16 \mathrm{~mm}\left(0.31 \lambda_{0}\right)$ & $w_{s}$ & $0.3 \mathrm{~mm}\left(0.0058 \lambda_{0}\right)$ \\
\hline $\mathrm{L}_{\mathrm{y}}$ & $11 \mathrm{~mm}\left(0.21 \lambda_{0}\right)$ & $2 r$ & $2.0 \mathrm{~mm}\left(0.0386 \lambda_{0}\right)$ \\
\hline
\end{tabular}

TABLE II

Electrical Characteristics of a CONVEntional SBA And the Proposed SBA (AT 5.8 GHz)

\begin{tabular}{|l|c|c|}
\hline & Conventional SBA & Proposed SBA \\
\hline Effective gain (dBi) & 14.3 & 14.3 \\
\hline Half power beam width (degrees) & $34^{\circ}$ & $34^{\circ}$ \\
\hline Directive gain (dBi) & 14.8 & 14.5 \\
\hline $1^{\text {st }}$ sidelobe level (dB) & -21.0 & -20.0 \\
\hline Axial ratio (dB) & 1.28 & 1.5 \\
\hline Aperture efficiency with respect to & $65 \%$ & $72 \%$ \\
Effective gain & $73 \%$ & $75 \%$ \\
Directive gain & $3 \%(V S W R \leq 1.5)$ & $6.5 \%(V S W R \leq 1.2)$ \\
\hline Frequency bandwidth for VSWR & & \\
\hline
\end{tabular}

by numerous simulations. The optimization was performed by applying a general design guideline as follows: 1) Choose the diameters of the primary reflector and the subreflector $\left(D_{r}\right.$ and $\left.D_{s}\right)$, and the height of the subreflector $\left(H_{r}\right)$ initially to be the same as those of a conventional circularly polarized SBA [6], namely, $D_{r}=2.0 \lambda_{0}, D_{s}=0.46 \lambda_{0}, H_{r}=0.5 \lambda_{0}$ (= the height of the rim); 2) Let the slotted patch have the same size as the subreflector and set its height $\left(H_{s}\right)$ initially to $0.25 \lambda_{0}$ ; 3) Design two orthogonal H-shaped slots, feed probe, and the shorting pin based on the geometric parameters presented in [11]; 4) Reduce the length of one of the H-shaped slots for achieving circularly polarized radiation; 5) Introduce a pair of capacitive stubs in the longer $\mathrm{H}$-shaped slot and adjust the geometry of the H-shaped slots (i.e., $W_{w}, W_{n}, L_{w}, L_{x}$, and $L_{y}$ ) for a desirable performance for circular polarization; 6) Adjust the position and diameter of the feed probe and shorting pin for impedance matching; 7) Optimize the overall size of the SBA for the best radiation (i.e., lowest axial ratio in $\mathrm{dB}$ with highest gain) and impedance matching performance.

\section{Results}

Fig. 2 shows the simulated and measured results for VSWR of the cross-aperture-excited SBA. Good agreement is observed. The bandwidth for VSWR $<1.2$ is found to be about $6.5 \%$, much better than the conventional cross-dipole-excited SBA [6]. The axial ratio (AR) at broadside (in the $z$ direction) and gain are plotted in Fig. 3. It is seen that the bandwidth for $A R \leqslant 3 \mathrm{~dB}$ is about $4.2 \%$ and the gain is around $14 \mathrm{dBi}$. The aperture efficiency is $70-80 \%$. The radiation pattern measured at $5.8 \mathrm{GHz}$ is compared with the simulated result in Fig. 4 and good agreement is observed for the co-polarization (i.e., the left-hand circular polarization, LHCP) over the main beam. The half power beam width is about $35^{\circ}$ and the first sidelobe level is less than

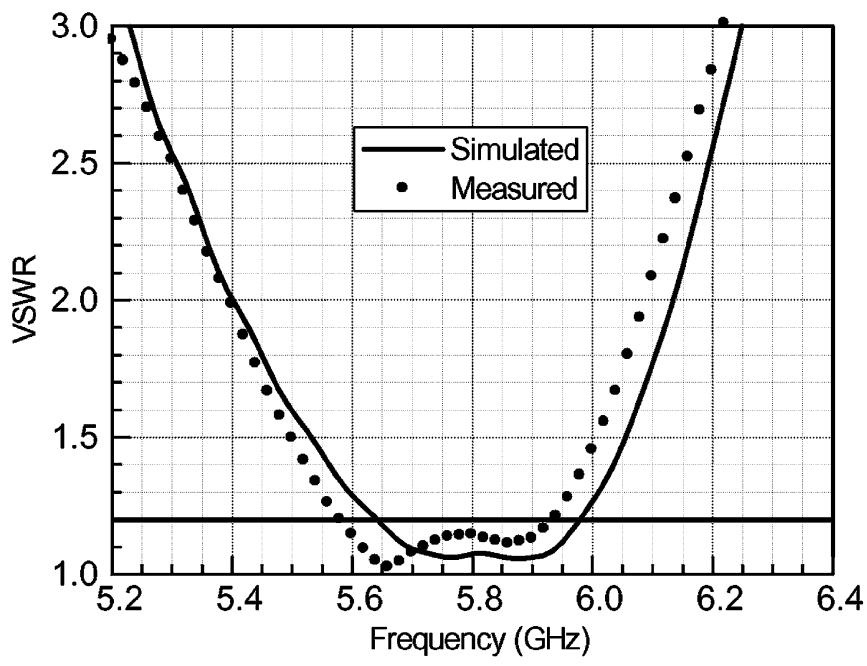

Fig. 2. Simulated and measured results for VSWR of the cross-apertureexcited SBA.

$-20 \mathrm{~dB}$. The cross-polarization (i.e., the right-hand circular polarization, RHCP) is less than $-15 \mathrm{~dB}$. The radiation characteristics of the cross-aperture-excited SBA are comparable to those of a cross-dipole-excited SBA [6]. A more detailed comparison of the electrical characteristics between a conventional SBA [6] and the proposed SBA is shown in Table II.

\section{ANALYSIS}

The unbalanced feed of the cross-aperture-excited SBA can be decomposed into two distinct driving modes, namely, an even mode and an odd mode [11]. Since the even-mode impedance is usually higher than the odd-mode impedance around the design frequency, the unbalanced input impedance is dominated by the odd-mode impedance. It is also noted that for the even mode 


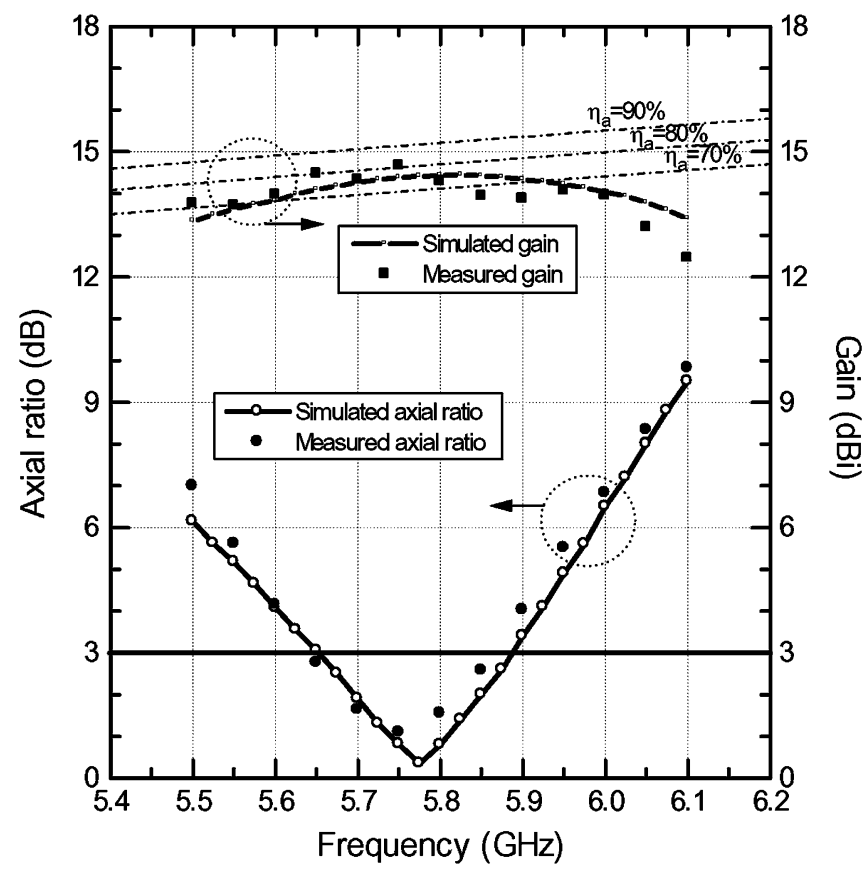

Fig. 3. Simulated and measured results for axial ratio and gain of the crossaperture-excited SBA.

the excitation structure is essentially a cap-loaded monopole, which does not contribute to the radiation at broadside. Therefore we only need to focus on the odd mode that looks like a balanced center feed through a two-wire (the feed probe plus the shorting pin) transmission line. For a diagonally-aligned center feed (simply called "diagonal-feed"), the voltage source $V_{0}$ can be divided into two voltage components $V_{x}$ (called "X-feed") and $V_{y}$ ("Y-Feed"), which cross Slots $\mathrm{X}$ and $\mathrm{Y}$ respectively, as illustrated in Fig. 5. The input impedance $\left(Z^{\circ}\right)$ of the diagonal-feed simply equals the summation of the input impedances $\left(Z_{x}\right.$ and $Z_{y}$ ) of the $\mathrm{X}$-feed and the Y-feed, that is

$$
Z^{\circ}=Z_{x}+Z_{y}
$$

The input impedance $Z^{\circ}$ of the diagonal-feed is transformed into the odd-mode impedance $Z_{\text {in }}^{o}$ through the two-wire transmission line. Therefore we can obtain

$$
Z_{\text {in }}^{o}=Z_{c} \frac{Z^{o}+j Z_{c} \tan \beta H_{s}}{Z_{c}+j Z^{o} \tan \beta H_{s}}
$$

where $\beta=2 \pi / \lambda_{0}, H_{s}$ is the length of the transmission line (or the height of the slotted patch), and $Z_{c}$ is the characteristic impedance of the two-wire transmission line. Fig. 6 shows $Z_{x}$ and $Z_{y}$ simulated by directly enforcing a voltage source across the slot and compares the calculated odd-mode impedance $Z_{\text {in }}^{o}$ with the simulated and measured input impedance $Z_{\text {in }}$. In the simulation for $Z_{x}$ and $Z_{y}$, the feed probe and the shorting pin were removed and a wire of radius $0.05 \mathrm{~mm}$ was used to connect the voltage source across the slot. A more detailed description for the calculation of $Z_{x}$ and $Z_{y}$ is presented in Appendix.

It is seen that the calculated $Z_{\text {in }}^{o}$ is close to $Z_{\text {in }}$. The difference between $Z_{\text {in }}^{o}$ and $Z_{\text {in }}$ is due to the contribution from the even mode and due to the approximation of the feeding structure introduced during the simulation (such as the wire

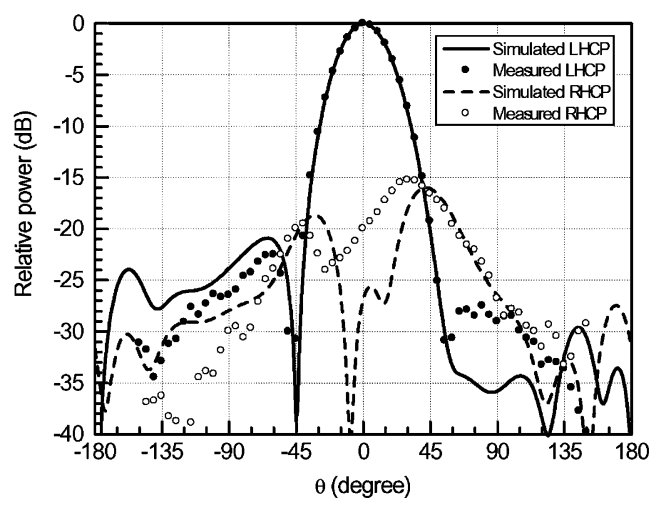

(a)

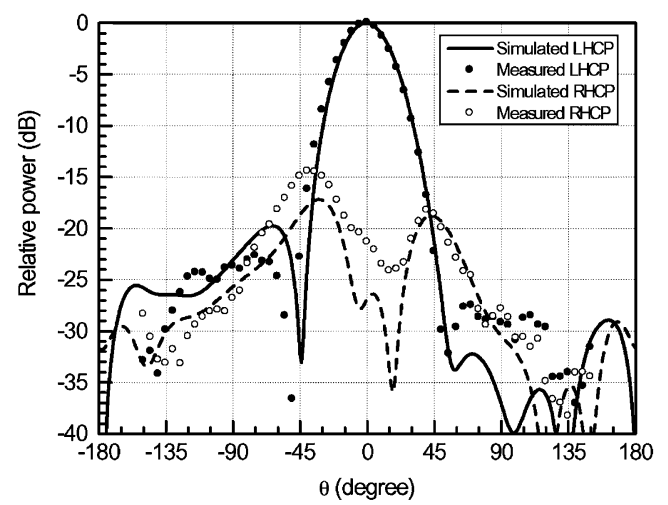

(b)

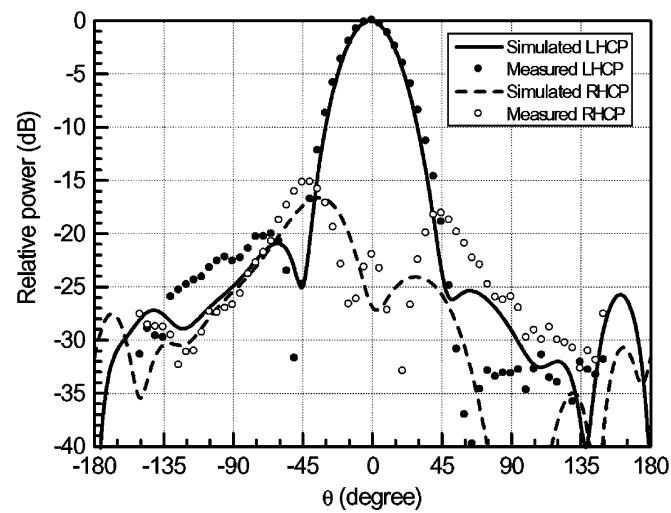

(c)

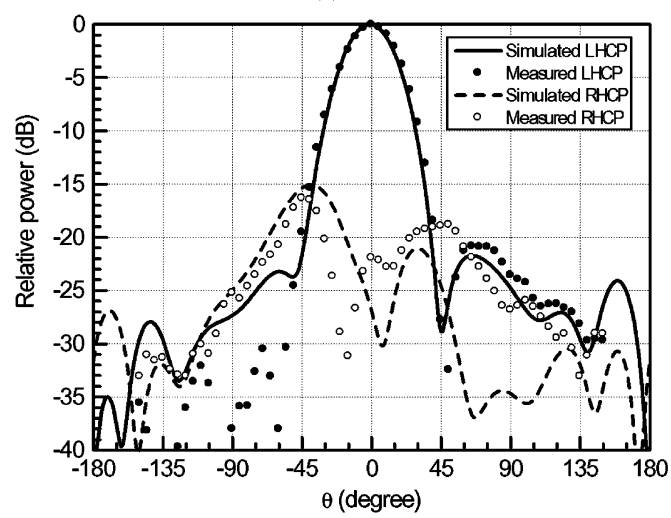

(d)

Fig. 4. Simulated and measured radiation patterns of the cross-apertureexcited SBA (at $5.8 \mathrm{GHz}$ ). (a) $\phi=0^{\circ}$. (b) $\phi=45^{\circ}$. (c) $\phi=90^{\circ}$. (d) $\phi=135^{\circ}$.

introduced to connect the voltage source). It is interesting to note that the two-wire transmission line formed by the feed 

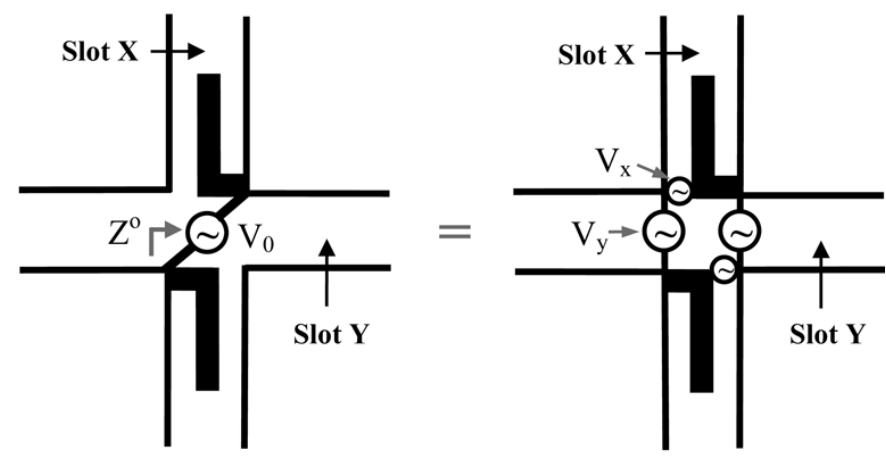

Diagonal-Feed
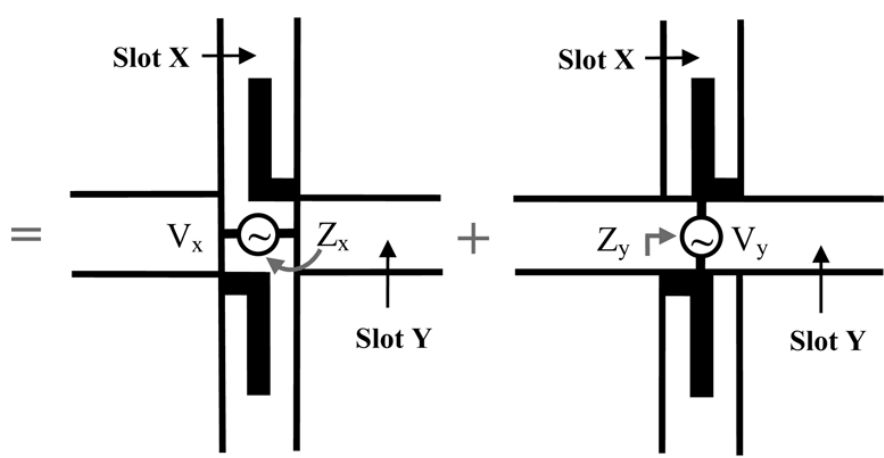

X-Feed

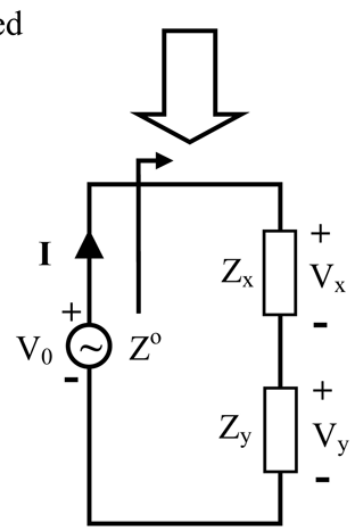

Equivalent circuit

Fig. 5. The diagonal-feed $\left(V_{0}\right)$ of a cross aperture is considered as a sum of an X-feed (crossing Slot $\mathrm{X}, V_{x}$ ) and a Y-feed (crossing Slot $\mathrm{Y}, V_{y}$ ).

probe and the shorting pin can transform the high-value diagonal-fed input impedance $Z^{\circ}$ (about several hundreds of ohms) to a lower resistance (around $50 \mathrm{ohms}$ ). (Virtually the two-wire transmission line can be thought of as a quarter-wave transformer since its length is close to $\lambda_{0} / 4$.) Therefore the unbalanced feed serves as an impedance matching circuit as well as a transmission line. The impedance matching can be achieved by properly selecting the diameter and the center-to-center distance of the feed probe and the shorting pine and by adjusting the height of the slotted patch. A more detailed parametric study on the impedance matching can be found in [11].

Since the gain of an SBA is dominantly determined by the primary reflector, the subreflector, and the rim (not sensitive to the size of the excitation structure), the far-zone fields ( $E_{x}$ and $E_{y}$ ) generated at broadside by the $\mathrm{X}$-feed $\left(V_{x}\right)$ and the $\mathrm{Y}$-feed

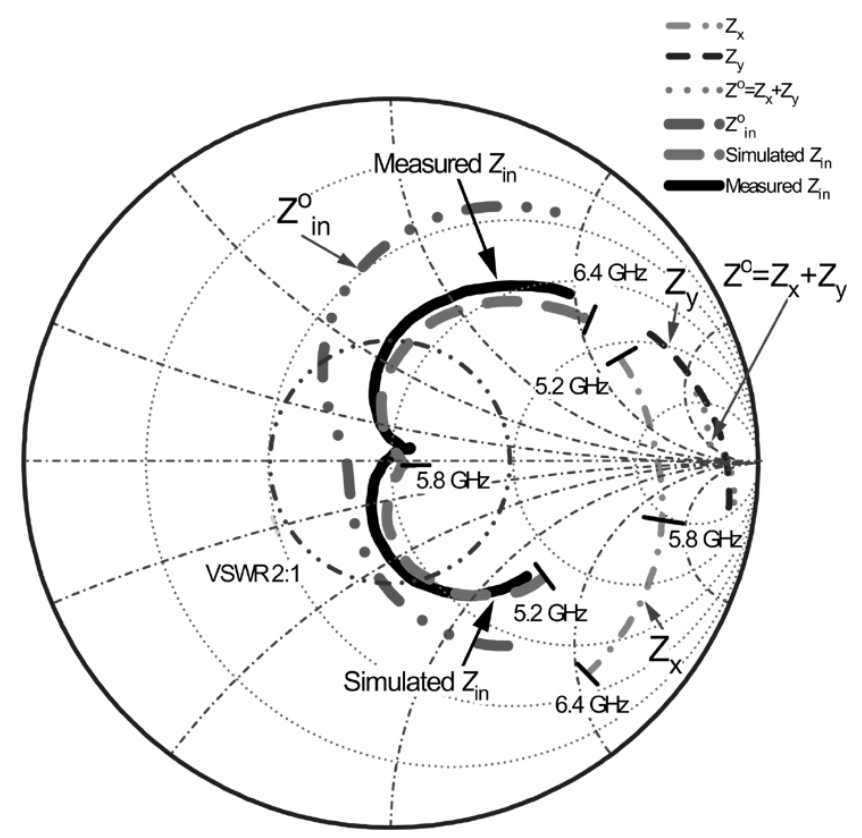

Fig. 6. Simulated and measured input impedances $\left(Z_{\text {in }}\right)$ of the cross-aperture-excited SBA are compared with the odd-mode impedance $\left(Z_{\text {in }}^{o}\right)$ calculated from $Z_{x}$ and $Z_{y}$.

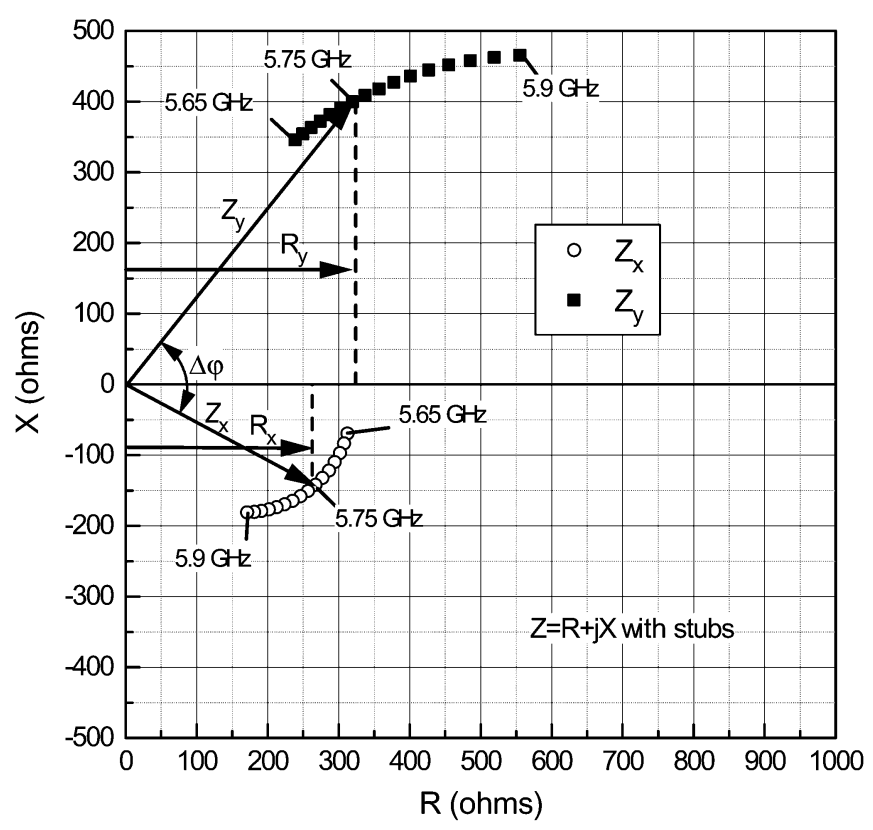

Fig. 7. $R-X$ diagram of the input impedances $Z_{x}$ and $Z_{y}$ for the $\mathrm{X}$-feed and the Y-feed with a pair of capacitive stubs.

$\left(V_{y}\right)$ are proportional to the square root of their input powers $\left(P_{x}\right.$ and $\left.P_{y}\right)$, that is

$$
\frac{\left|E_{x}\right|}{\left|E_{y}\right|}=\left(\frac{P_{x}}{P_{y}}\right)^{1 / 2}=\left(\frac{I^{2} R_{x}}{I^{2} R_{y}}\right)^{1 / 2}=\left(\frac{R_{x}}{R_{y}}\right)^{1 / 2}
$$

where $R_{x}$ and $R_{y}$ are the radiation resistances associated with the $\mathrm{X}$-feed and the Y-feed, respectively. (It is observed by simulation that the length of each slot has little effect on the amplitude pattern of the SBA.) The phase difference (denoted by $\Delta \varphi$ ) between $E_{x}$ and $E_{y}$ is associated with the excitations, i.e., $V_{x}$ and $V_{y}$, or the input impedances $Z_{x}$ and $Z_{y}$ of the $\mathrm{X}$-feed and 


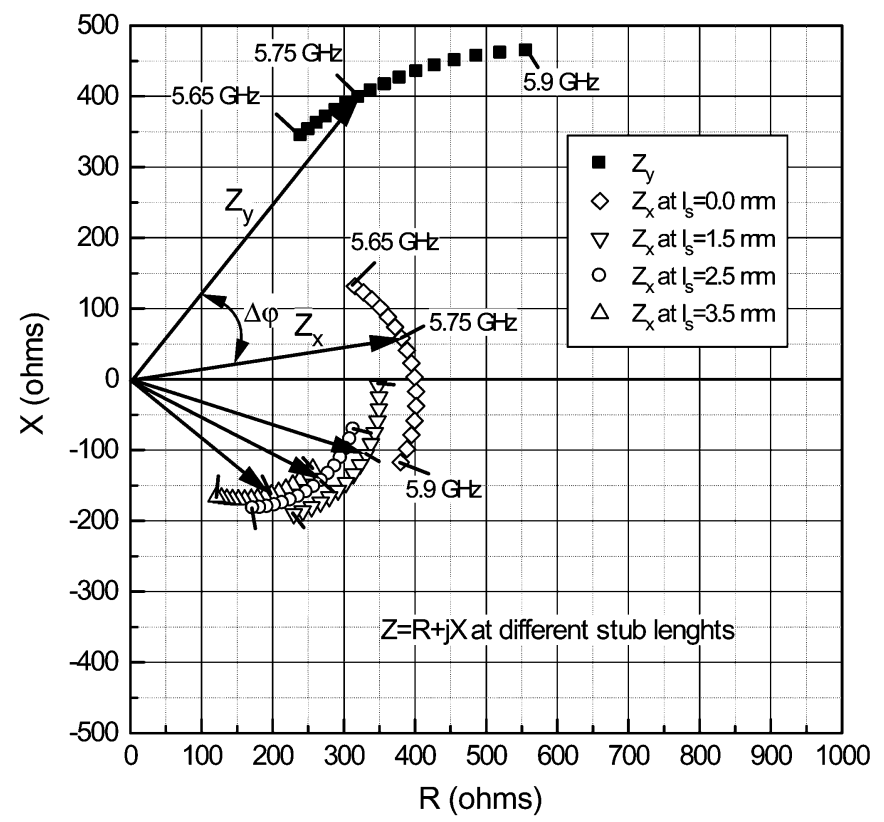

Fig. 8. Effect of the stub length $\left(l_{s}\right)$ on the input impedances $Z_{x}$ and $Z_{y}$ for the $\mathrm{X}$-feed and the Y-feed.

the Y-feed, respectively. For a perfectly circular polarization, it is required that $\left|E_{x}\right| /\left|E_{y}\right|=1$ (or $R_{x}=R_{y}$ ) and $\Delta \varphi= \pm 90^{\circ}$. Fig. 7 shows the $R-X$ diagram (the real part $R$ versus the imaginary part $X$ of an input impedance $Z$ ) for the impedances $Z_{x}$ and $Z_{y}$. As an example, looking at a frequency (say $5.75 \mathrm{GHz}$ ) in the $5.8 \mathrm{GHz}$ ISM band, it is found that the phase difference between $Z_{x}$ and $Z_{y}$ is near $-80^{\circ}$ (close to $\Delta \varphi=-90^{\circ}$ ) and that $\left(R_{x} / R_{y}\right)^{1 / 2}$ is equal to 0.91 (close to 1 ), which corresponds to an LHCP wave. Using $\left|E_{x}\right| /\left|E_{y}\right|=0.91$ and $\Delta \varphi=-80^{\circ}$, we can obtain an axial ratio of $1.8 \mathrm{~dB}$, close to the simulated and measured values (about $1.2 \mathrm{~dB}$ ).

Fig. 8 demonstrates the effect of the stub length $\left(l_{s}\right)$ on the impedances $Z_{x}$ and $Z_{y}$. Since $Z_{y}$ is decided by the field distribution in Slot $Y$ where there is no stub, the stub length almost has no effect on $Z_{y}$. Both the real part $\left(R_{x}\right)$ and the imaginary part $\left(X_{x}\right)$ of $Z_{x}$ vary with $l_{s}$. As $l_{s}$ increases, the phase difference $(\Delta \varphi)$ between $Z_{x}$ and $Z_{y}$ increases, but $R_{x}$ decreases. This is because a longer stub tends to blocking the radiation from Slot $\mathrm{X}$ while making Slot $\mathrm{X}$ become more capacitive (i.e., $X_{x}$ decreases). But if without a pair of capacitive stubs (i.e., $l_{s}=0$ ), $\Delta \varphi$ becomes much less than $90^{\circ}$ (only about $42^{\circ}$ ) even though $\left(R_{x} / R_{y}\right)^{1 / 2}$ is still close to 1 . Therefore the pair of capacitive stubs is considered to be essential for the production of circular polarization and there is an optimal value for $l_{s}$. By simulation and experiment, the optimized $l_{s}$ is found to be $2.5 \mathrm{~mm}$. The stub width $\left(w_{s}\right)$ is not critical for the achievement of circularly polarized radiation, but it is limited by the fabrication process [e.g., a printed circuit broad (PCB) technology], especially for a higher-frequency application. Usually, a PCB fabricator has a similar limitation on the minimum line width and slot width (i.e., the line to line clearance). Therefore, we selected $w_{s}$ to be $\sim W_{n} / 3$, which makes the minimum line width approximately equal the minimum line to line clearance. Fig. 8 also suggests that the performance for circular polarization will not significantly change if the stub length $\left(l_{s}\right)$ varies within $2.5 \pm 0.5 \mathrm{~mm}$.

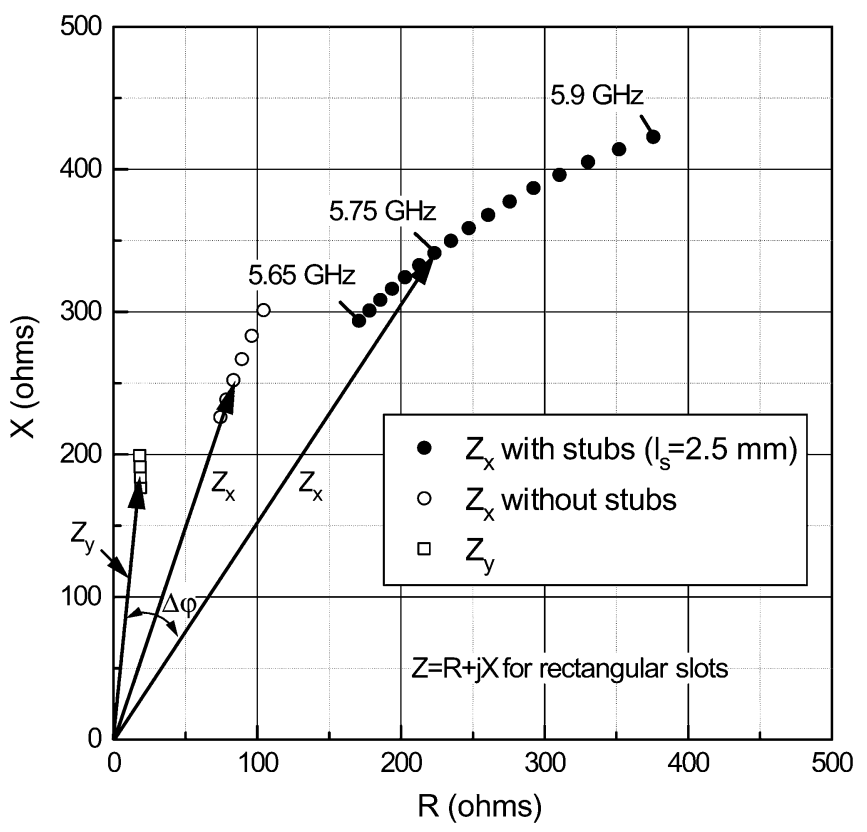

Fig. 9. $R-X$ diagram of the input impedances $Z_{x}$ and $Z_{y}$ for two rectangular slots $\left(W_{w}=W_{n}=1.0 \mathrm{~mm}\right)$.

Simulation shows that an error of $\pm 0.5 \mathrm{~mm}$ for $l_{s}$ or $\pm 0.1 \mathrm{~mm}$ for $w_{s}$ leads to an increase in the minimum axial ratio of $\sim 0.5 \mathrm{~dB}$.

Fig. 9 exhibits the $R-X$ diagram for two orthogonal rectangular slots whose lengths are equal to the total lengths of the H-shaped slots (i.e., $L_{w}+L_{x}$ for Slot X and $L_{w}+L_{y}$ for Slot Y). It clearly shows that both the amplitude [i.e., $\left(R_{x} / R_{y}\right)^{1 / 2}$ ] and the phase difference $(\Delta \varphi)$ are far from the requirements for circular polarization no matter whether there is a pair of capacitive stubs or not. So we conclude that it is difficult to achieve circular polarization with rectangular slots.

Finally it should be mentioned that the bandwidth of circular polarization (i.e., the axial ratio) is limited by the leaky cavity structure of the SBA not just by the excitation topology. By simulation, we find that the excitation topology does not have a significant effect on the AR bandwidth.

\section{CONCLUSION}

A cross aperture has been proposed to excite the short backfire antenna (SBA) for circular polarization. The cross aperture consists of two orthogonal $\mathrm{H}$-shaped slots fed by a single probe with a shorting pin. The feed probe and the shorting pin form an unbalanced feed which serves as a two-wire transmission line and acts as an impedance matching circuit. A pair of capacitive stubs is introduced inside the cross aperture to enhance the capacitance of one of the H-shaped slot. It has been demonstrated that the H-shaped slots and the capacitive stubs are essential for the achievement of circular polarization. The cross-aperture-excited SBA has the advantage of no need for a balun circuit and for an external polarizer. The proposed SBA achieves an impedance bandwidth of $6.5 \%$ (VSWR $<1.2$ ), an axial ratio $(\leqslant 3 \mathrm{~dB})$ bandwidth of $4.2 \%$, and a gain of $14 \mathrm{dBi}$. Simulation and experimental results show good agreement. 


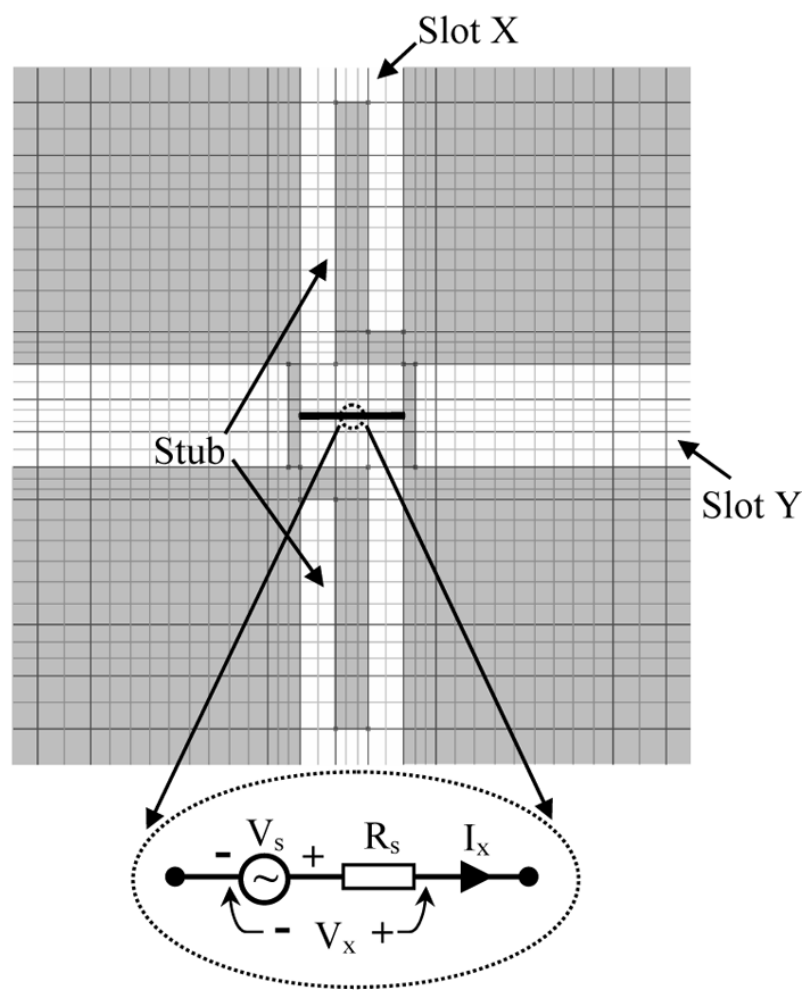

Wire port

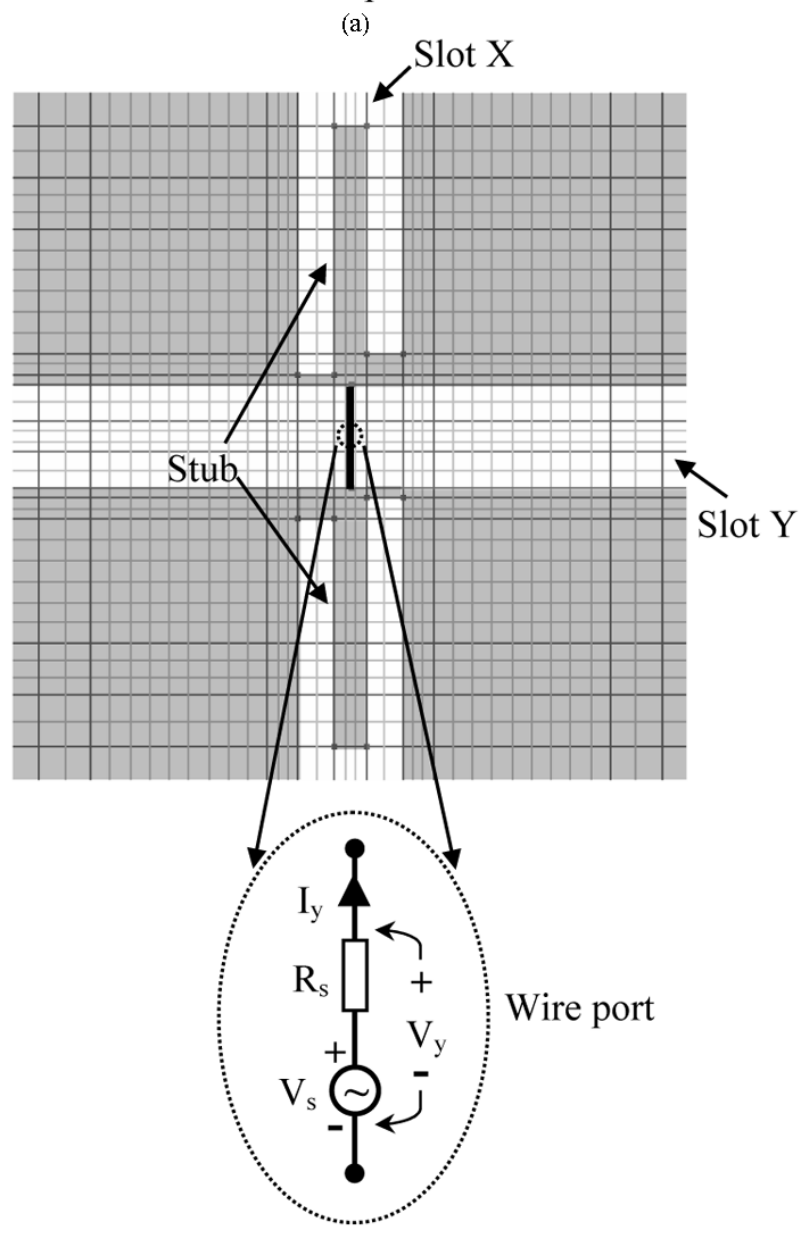

(b)

\section{APPENDIX \\ CALCUlation of $Z_{x}$ and $Z_{y}$}

The impedances $Z_{x}$ and $Z_{y}$ are calculated using MicroStripes V6.5 which is a time-domain electromagnetic solver based on the transmission-line matrix (TLM) method [12]. The TLM mesh around the center of the cross aperture is illustrated in Fig. 10. For the calculation of $Z_{x}$ and $Z_{y}$ a wire port is introduced across the slot $\mathrm{X}$ and slot $\mathrm{Y}$, respectively. The wire port is formed with a voltage source which consists of the source voltage $V_{s}$ and the internal source resistance $R_{s}$. Through the TLM simulation, we can obtain the wire current ( $I_{x}$ for $Z_{x}$ or $I_{y}$ for $Z_{y}$ ) and the wire voltage $\left(V_{x}\right.$ for $Z_{x}$ or $V_{y}$ for $Z_{y}$ ), from which the impedances $Z_{x}$ and $Z_{y}$ are determined as $Z_{x}=V_{x} / I_{x}$ and $Z_{y}=V_{y} / I_{y}$.

\section{ACKNOWLEDGMENT}

The authors wish to thank the Reviewers for their valuable suggestions.

\section{REFERENCES}

[1] H. W. Ehrenspeck, "The short-backfire antenna," Proc. IEEE, vol. 53, no. 6, pp. 1138-1140, Aug. 1965.

[2] A. Kumar and H. D. Hristov, Microwave Cavity Antennas. Norwood, MA: Artech House, 1989, pp. 215-386.

[3] Mobile Antenna Systems Handbook, 2nd ed., K. Fujimoto and J. R. James, Eds., Artech House, Norwood, MA, 2000, pp. 542-545.

[4] K. M. Keen, "Feeder errors cause antenna circular polarization deterioration," Microwave System News, vol. 14, no. 5, pp. 102-108, May 1984.

[5] R. J. Katulski, "The optimization of the crossed dipoles as a radiating source in the short back fire antenna," in Proc. 3rd Int. Conf. Antenna and Communications, ICAP'83, IEE Conf. Pub., no. 219. London, U.K., 1983, pp. 412-416.

[6] S. Ohmori, S. Miura, K. Kameyama, and H. Yoshimura, "An improvement in electrical characteristics of a short backfire antenna," IEEE Trans. Antennas Propag., vol. 31, no. 4, pp. 644-646, Jul. 1983.

[7] H. Iwasaki, "A circularly polarized small-size microstrip antenna with a cross slot," IEEE Trans. Antennas Propag., vol. 44, no. 10, pp. 1399-1401, Oct. 1996.

[8] C.-Y. Huang, J.-Y. Wu, and K.-L. Wong, "Cross-slot-coupled microstrip antenna and dielectric resonator antenna for circular polarization," IEEE Trans. Antennas Propag., vol. 47, no. 4, pp. 605-609, Apr. 1999.

[9] B. Al-Jihouri, H. Evans, E. Korolkiewicz, E. G. Lim, A. Sambell, and T. Viasits, "Cavity model of circularly polarized cross-aperture-coupled microstrip antenna," Proc. Inst. Elect. Eng.-Micro. Antennas Propag., vol. 148, no. 3, pp. 147-152, Jun. 2001.

[10] D. Sievenpiper, H.-P. Hsu, and R. M. Riley, "Low-profile cavity-backed crossed-slot antenna with a single-probe feed designed for $2.34-\mathrm{GHz}$ satellite radio applications," IEEE Trans. Antennas Propag., vol. 52, no. 3, pp. 873-879, Mar. 2004

[11] R. L. Li, D. Thompson, M. M. Tentzeris, J. Laskar, and J. Papapolymerou, "Development of a wideband short backfire antenna excited by an unbalance-fed H-shaped slot," IEEE Trans. Antenna and Propagat., vol. 53, no. 2, pp. 662-671, Feb. 2005.

[12] Micro-Stripes Reference Manual: Issue 1, 2004. Flomerics Ltd., U.K.

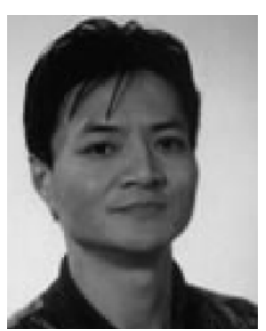

RongLin Li (M'02-SM'03) received the B.S. degree in electrical engineering from Xi' an Jiaotong University, China, in 1983 and the M.S. and Ph.D. degrees in electrical engineering from Chongqing University, China, in 1990 and 1994, respectively.

From 1983 to 1987, he was an Electrical Engineer with the Yunnan Electric Power Research Institute. From 1994 to 1996, he was a Postdoctoral Research Fellow with Zhejiang University, China. In 1997, he was with Hosei University, Japan, as a Hosei International Fund Research Fellow. Since 1998, he has been a Professor with Zhejiang University. In 1999, he was a Research Associate with the University of Utah. In 2000, he was a Research Fellow with Queen's University of Belfast, U.K. In 2001, he joined the ATHENA group as a Research Scientist with the Georgia Institute of Technology, Atlanta. His latest research interests include computational electromagnetics, modeling of antennas and microwave devices, and RF packaging design. 


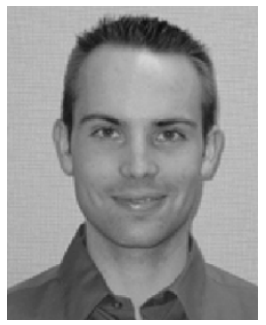

Dane C. Thompson (S'98) was born in Sacramento, CA, in February 1979. He received the B.S. and M.S. degrees in electrical engineering from Santa Clara University, Santa Clara, CA, in 2001 and 2002 respectively, and is currently working toward the $\mathrm{Ph} . \mathrm{D}$. degree in electrical and computer engineering at the Georgia Institute of Technology, Atlanta.

His research involves the processing and use of LCP as a high-performance dielectric substrate and packaging material. He is currently researching the utilization of LCP for vertically integrated RF front-ends, for MMIC and MEMS packaging, and for dual-frequency dual-polarization multilayer conformal antennas.

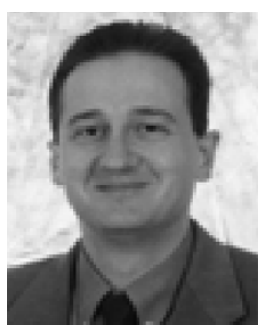

John Papapolymerou (S'90-M'99-SM'04) received the B.S.E.E. degree from the National Technical University of Athens, Athens, Greece, in 1993, and the M.S.E.E. and Ph.D. degrees from the University of Michigan, Ann Arbor, in 1994 and 1999, respectively.

From 1999 to 2001, he was a faculty member at the Department of Electrical and Computer Engineering of the University of Arizona, Tucson, and during summers 2000 and 2003, he was a Visiting Professor at The University of Limoges, France. From 2001 to 2005 he was an Assistant Professor at the School of Electrical and Computer Engineering, Georgia Institute of Technology, Atlanta, where he is currently an Associate Professor. His research interests include the implementation of micromachining techniques and MEMS devices in microwave, millimeter-wave and $\mathrm{THz}$ circuits and the development of both passive and active planar circuits on semiconductor $(\mathrm{Si} / \mathrm{SiGe}, \mathrm{GaAs})$ and organic substrates (LCP, LTCC) for System-on-a-Chip (SOC)/ System-on-a-Package (SOP) RF front ends. He has authored or coauthored over 120 publications in peer reviewed journals and conferences.

Dr. Papapolymerou received the 2004 Army Research Office (ARO) Young Investigator Award, the 2002 National Science Foundation (NSF) CAREER award, the best paper award at the 3rd IEEE International Conference on Microwave and Millimeter-Wave Technology (ICMMT2002), Beijing, China and the 1997 Outstanding Graduate Student Instructional Assistant Award presented by the American Society for Engineering Education (ASEE), The University of Michigan Chapter. His student also received the best student paper award at the 2004 IEEE Topical Meeting on Silicon Monolithic Integrated Circuits in RF Systems, Atlanta, GA. He currently serves as the Vice-Chair for Commission D of the US National Committee of URSI and as an Associate Editor for IEEE TRansactions on Antennas and Propagation. During 2004 he was the Chair of the IEEE MTT/AP Atlanta Chapter.

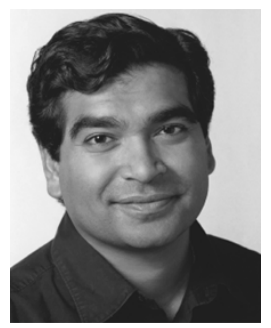

Joy Laskar (S'84-M'85-SM'02-F'05) received the B.S. degree in computer engineering (math/physics minors, highest honors) from Clemson University, Clemson, SC, in 1985 and the M.S. and Ph.D. degrees in electrical engineering from the University of Illinois, Urbana-Champaign, in 1989 and 1991, respectively.

Prior to joining the Georgia Institute of Technology (Georgia Tech), Atlanta, in 1995, he held faculty positions at the University of Illinois and the University of Hawaii. At Georgia Tech, he holds the Joseph M. Pettit Professorship of Electronics, is currently the Chair for the Electronic Design and Applications Technical Interest Group, the Director of
Georgia's Electronic Design Center and the System Research Leader for the NSF Packaging Research Center. In 1998, he cofounded an advanced WLAN IC Company: RF Solutions, which is now part of Anadgics (Nasdaq: Anad). In 2001, he cofounded a next generation interconnect company: Quellan, which is developing collaborative signal processing solutions for enterprise applications. He also heads a research group with a focus on integration of high-frequency electronics with optoelectronics and integration of mixed technologies for next generation wireless and optoelectronic systems. His research has focused on high frequency IC design and their integration. He has authored or coauthored more than 200 papers, several book chapters (including three textbooks in development), numerous invited talks, and has 10 patents pending.

Dr. Laskar is a 1995 recipient of the Army Research Office's Young Investigator Award, a 1996 recipient of the National Science Foundation's CAREER Award, the 1997 NSF Packaging Research Center Faculty of the Year, the 1998 NSF Packaging Research Center Educator of the Year, the 1999 corecipient of the IEEE Rappaport Award (Best IEEE Electron Devices Society Journal Paper), the faculty advisor for the 2000 IEEE MTT IMS Best Student Paper award, 2001 Georgia Tech Faculty Graduate Student Mentor of the year, recipient of a 2002 IBM Faculty Award, the 2003 Clemson University College of Engineering Outstanding Young Alumni Award and the 2003 recipient of the Outstanding Young Engineer of the Microwave Theory and Techniques Society. He has been named the Joseph M. Pettit Professor of Electronics in the School of Electrical and Computer Engineering at Georgia Tech. For the 2004-2006 term, he has been appointed an IEEE Distinguished Microwave Lecturer for his seminar entitled "Recent Advances in High Performance Communication Modules and Circuits."

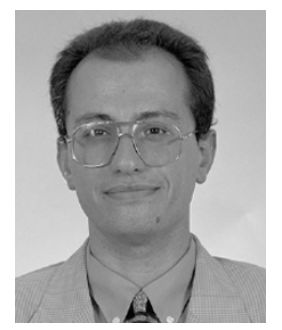

Manos M. Tentzeris (S'89-M'98-SM'03) received the diploma degree in electrical and computer engineering (magna cum laude) from the National Technical University of Athens, Greece, and the M.S. and $\mathrm{Ph} . \mathrm{D}$. degrees in electrical engineering and computer science from the University of Michigan, Ann Arbor

He was a Visiting Professor with the Technical University of Munich, Germany for the summer 2002, where he introduced a course in the area of high-frequency packaging. He is currently an Associate Professor with the School of Electrical and Computer Engineering, Georgia Institute of Technology (Georgia Tech), Atlanta. He has helped develop academic programs in highly integrated/multilayer packaging for RF and wireless applications, microwave MEMs, SOP-integrated antennas and adaptive numerical electromagnetics (FDTD, multiresolution algorithms) and heads the ATHENA research group (15 researchers). He is the Georgia Tech NSF-Packaging Research Center Associate Director for RF Research and the RF Alliance Leader. He is also the leader of the RFID Research Group of the Georgia Electronic Design Center (GEDC) of the State of Georgia. He has given more than 40 invited talks in the same area to various universities and companies in Europe, Asia and America. He has published more than 180 papers in refereed journals and conference proceedings, eight book chapters, and he is in the process of writing three books.

Dr. Tentzeris is a member of the International Scientific Radio Union (URSI)Commission D, an Associate Member of EuMA, and a member of the Technical Chamber of Greece. He was the recipient of the 1997 Best Paper Award of the International Hybrid Microelectronics and Packaging Society for the development of design rules for low-crosstalk finite-ground embedded transmission lines. He received the 2000 NSF CAREER Award for his work on the development of MRTD technique that allows for the system-level simulation of RF integrated modules, the 2001 ACES Conference Best Paper Award, the 2002 International Conference on Microwave and Millimeter-Wave Technology Best Paper Award (Beijing, China) for his work on Compact/SOP-integrated RF components for low-cost high-performance wireless front-ends, the 2002 Georgia Tech-ECE Outstanding Junior Faculty Award, the 2003 NASA Godfrey "Art" Anzic Collaborative Distinguished Publication Award for his activities in the area of finite-ground low-loss low-crosstalk coplanar waveguides, the 2003 IBC International Educator of the Year Award, the 2003 IEEE CPMT Outstanding Young Engineer Award for his work on 3-D multilayer integrated RF modules, and the 2004 IEEE Transactions on Advanced Packaging Commendable Paper Award. He was also the 1999 Technical Program Co-Chair of the 54th ARFTG Conference, Atlanta, GA and he is the Vice-Chair of the RF Technical Committee (TC16) of the IEEE CPMT Society. He has organized various sessions and workshops on RF/Wireless Packaging and Integration in IEEE ECTC, IMS and APS Symposia in all of which he is a member of the Technical Program Committee in the area of "Components and RF." He is an Associate Editor of the IEEE TRANSACTIONS ON ADVANCED PACKAGING. 